\title{
Experimental Evidence for a State-Point-Dependent Density-Scaling Exponent of Liquid Dynamics
}

\author{
Alejandro Sanz, ${ }^{*}$ Tina Hecksher, Henriette Wase Hansen, Jeppe C. Dyre, Kristine Niss, and Ulf R. Pedersen ${ }^{\dagger}$ \\ Glass and Time, IMFUFA, Department of Science and Environment, Roskilde University, \\ P.O. Box 260, DK-4000 Roskilde, Denmark
}

(Received 13 August 2018; published 8 February 2019)

\begin{abstract}
A large class of liquids obey density scaling characterized by an exponent, which quantifies the relative roles of temperature and density for the dynamics. We present experimental evidence that the densityscaling exponent $\gamma$ is state-point dependent for the glass formers tetramethyl-tetraphenyl-trisiloxane (DC704) and 5-polyphenyl ether (5PPE). A method is proposed that from dynamic and thermodynamic properties at equilibrium estimates the value of $\gamma$. The method applies at any state point of the pressuretemperature plane, both in the supercooled and the normal liquid regimes. We find that $\gamma$ is generally statepoint dependent, which is confirmed by reanalyzing data for 20 metallic liquids and two model liquids.
\end{abstract}

DOI: 10.1103/PhysRevLett.122.055501

Decreasing the temperature $(T)$ or increasing the density $(\rho)$ by applying pressure to a liquid leads to a slowing down of the molecular dynamics and eventually to a glass transition if crystallization is avoided [1,2]. It has been demonstrated that for many low-molecular weight liquids and polymers, the relaxation time and other dynamic quantities can be superimposed onto master curves within the experimental uncertainty when plotted as a function of $\rho^{\gamma} / T$ [3]. The density-scaling exponent $\gamma$ is often regarded as a material constant [4-6]. Indeed, recent experiments have shown that in certain cases $\gamma$ is constant over a huge pressure range $[7,8]$. In this Letter we show, however, that $\gamma$ for other systems is not constant but state-point dependent; this is done by using a novel formalism that allows $\gamma$ to be determined from properties exclusively referring to the state point in question.

In the following we use subscripts to distinguish between different $\gamma$ 's. Let $\tau$ be the structural relaxation time determined, e.g., from the dielectric loss-peak frequency. We quote $\tau$ in reduced units (i.e., in units of $\sqrt{m \rho^{2 / 3} / k_{B} T}$ where $m$ is the atomic, molecular, or polymer-segment mass) and define the density-scaling exponent of $\tau$ as [9]

$$
\gamma_{\tau}(\rho, T) \equiv\left(\frac{\partial \log T}{\partial \log \rho}\right)_{\tau}
$$

We use base 10 logarithms. In this definition the exponent generally depends on the state point in question and the

Published by the American Physical Society under the terms of the Creative Commons Attribution 4.0 International license. Further distribution of this work must maintain attribution to the author(s) and the published article's title, journal citation, and DOI. quantity kept fixed, which is $\tau$ in the above case. The physical interpretation of $\gamma_{\tau}$ is that it quantifies the relative contributions of volume and temperature to the variation of $\tau$ in the thermodynamic phase diagram [10].

In analogy to the structural relaxation time, the configurational adiabats can also be associated with a densityscaling exponent:

$$
\gamma_{S_{\mathrm{ex}}}(\rho, T) \equiv\left(\frac{\partial \log T}{\partial \log \rho}\right)_{S_{\mathrm{ex}}}
$$

Here $S_{\text {ex }}$ is the entropy in excess of the ideal gas contribution $S_{\text {id }}: S_{\text {ex }} \equiv S-S_{\text {id }}$. In general, two density-scaling exponents of different observables (structural, dynamical, or thermodynamic) differ, e.g., $\gamma_{\tau} \neq \gamma_{S_{\text {ex }}}$. In Ref. [11] it was shown experimentally that $\gamma_{\tau}=\gamma_{S_{\mathrm{ex}}}$ at a state point of the silicone oil DC704, confirming a prediction of the isomorph theory [9] believed to apply to systems where van der Waals interactions dominate (but not to systems where hydrogen bonds dominate the Hamiltonian [12-14]).

The isomorph theory $[9,12-15]$ is a framework for describing systems where the potential energy function $U(\mathbf{R})$ possesses hidden scale invariance (here, $\mathbf{R}$ is the collective coordinate of the system). Hidden scale invariance can be formulated as the following criterion for two configurations $a$ and $b$ : if $U\left(\mathbf{R}_{a}\right)<U\left(\mathbf{R}_{b}\right)$ then $U\left(\lambda \mathbf{R}_{a}\right)<U\left(\lambda \mathbf{R}_{b}\right)$ to a good approximation where $\lambda>0$ is a scaling parameter [15]. For these systems, densityscaling exponents of many properties have the same statepoint dependence because they are all controlled by the excess entropy. Thus, there is just one density-scaling exponent, namely, the one of Eq. (2) that is given by the slope of the so-called isomorphs [9] along which dynamical, structural, and some thermodynamic properties are 
constant in reduced units. As an application, the isomorph theory explains Rosenfeld's excess entropy scaling law [16-19], i.e., the fact that the relaxation time in many systems is a function of the excess entropy: $\tau\left(S_{\mathrm{ex}}\right)$ [20].

In the isomorph theory, $\gamma$ generally depends on both density and temperature [15]. Previously, Alba-Simionesco, Kivelson and Tarjus (AKT) investigated the validity of a scaling law for activated dynamics where the density-scaling exponent is a function of only the density [2,21,22]. Regarding $\gamma$ as a material constant is an even more restrictive assumption, which only applies rigorously if the potential part of the Hamiltonian can be approximated by a sum of inverse power-law (IPL) pair interactions $r^{-n}$ plus an arbitrary constant (the "IPL hypothesis"; $n$ is the same for all terms but prefactors may differ) [4,23-27]. In that case, the density-scaling exponent is state-point independent and the relaxation time is simply a function of $\rho^{\gamma} / T$ with $\gamma=n / 3$.

In this Letter we investigate the state-point dependence of $\gamma$ without any model assumptions in a treatment that emphasizes error estimates. In general, the following four scenarios are possible: (A) $\gamma$ is a material constant (the IPL hypothesis), (B) $\gamma$ is a function only of density (the AKT hypothesis), (C) $\gamma$ varies throughout the two-dimensional thermodynamic phase diagram in matter controlled by the excess entropy (the isomorph-theory prediction [15]), or (D) the completely general case in which there are different density-scaling exponents for different quantities, each of which generally varies throughout the phase diagram.

We first give an expression for $\gamma_{\tau}$ in terms of quantities that can be measured at isobaric or isothermal conditions where most experiments are performed. Inspired by Angell [28] we define the following generalized fragility $[6,10,22,28-30]$ :

$$
m_{B}^{A} \equiv\left(\frac{\partial \log \tau}{\partial A}\right)_{B}
$$

where $A$ and $B$ are thermodynamic variables such as $T, \rho$, or $p$. Note that the generalized fragility is not defined as a dimensionless Angell-type index. Moreover, the temperature fragility $m_{p}^{T}$ (which is negative) is related to the apparent activation enthalpy [6] $H_{p} \equiv k_{B}[\partial \log \tau / \partial(1 / T)]_{p}$ by $H_{p}=-k_{B} T^{2} m_{p}^{T}$. When the temperature fragility $m_{p}^{T}$ is evaluated at the glass transition temperature $T_{g}$ at ambient pressure $(0.1 \mathrm{MPa})$, the relation to Angell's dimensionless fragility index [31] is given by $m_{\text {Angell }} \equiv$ $\left[\partial \log \tau / \partial\left(T_{g} / T\right)\right]_{p, T=T_{g}}=-T_{g} m_{p=0.1 \mathrm{MPa}}^{T=T_{g}}$. The isochoric activation energy $E_{V} \equiv k_{B}[\partial \log \tau / \partial(1 / T)]_{\rho}[6]$ can also be expressed via a generalized fragility as $E_{V}=-k_{B} T^{2} m_{\rho}^{T}$.

We next consider the pressure fragility $m_{T}^{p}$ and temperature fragility $m_{p}^{T}$ because these two quantities are directly experimentally accessible. We rewrite $\gamma_{\tau}$, Eq. (1), in terms of the ratio between two generalized fragilities by using the identity $(\partial \log T / \partial \log \rho)_{\tau}=$ $-(\partial \log \tau / \partial \log \rho)_{T}(\partial \log T / \partial \log \tau)_{\rho}$, resulting in

$$
\gamma_{\tau}=-\frac{\rho m_{T}^{\rho}}{T m_{\rho}^{T}}
$$

Typically the pressure and not the density is controlled in an experiment. Thus, we aim for an expression involving $m_{T}^{p}$ and $m_{p}^{T}$. From the chain rule one has $m_{T}^{\rho}=$ $(\partial \log \tau / \partial p)_{T}(\partial p / \partial \rho)_{T}$. It follows that

$$
m_{T}^{\rho}=K_{T} m_{T}^{p} / \rho,
$$

in which $K_{T} \equiv(\partial p / \partial \log \rho)_{T}$ is the isothermal bulk modulus. Using the identity $(\partial \log \tau / \partial T)_{\rho}=(\partial \log \tau / \partial T)_{p}+(\partial \log \tau /$ $\partial p)_{T}(\partial p / \partial T)_{\rho}$, and inserting the thermal-expansion coefficient at constant pressure $\alpha_{p}=-(\partial \log \rho / \partial T)_{p}$ one obtains

$$
m_{\rho}^{T}=m_{p}^{T}+\alpha_{p} K_{T} m_{T}^{p}
$$

Finally, by combining Eqs. (4)-(6) we arrive at an expression that relates $\gamma_{\tau}$ to directly measurable equilibrium properties

$$
\gamma_{\tau}=-\frac{K_{T} m_{T}^{p}}{T m_{p}^{T}+\alpha_{p} T K_{T} m_{T}^{p}} .
$$

Thus, the state-point dependence of the density-scaling exponent $\gamma_{\tau}$ can be obtained from the thermal-expansion coefficient $\left(\alpha_{p}\right)$, the pressure and temperature fragilities $\left(m_{T}^{p}\right.$ and $m_{p}^{T}$ ), and the isothermal bulk modulus $\left(K_{T}\right)$. The idea of computing $\gamma$ from two fragilities has been discussed previously in studies of supercooled liquids $[6,10,21,30]$. Here we have generalized this approach to the entire liquid state, i.e., also in the normal equilibrium liquid phase far from the glass transition.

Because of the measuring methods favored in our lab [32], we determine the isothermal bulk modulus from the adiabatic modulus $K_{S}$ measured from the speed of sound [32] via $K_{T}=K_{S} \rho C_{p} /\left[\rho C_{p}+T \alpha_{p}^{2} K_{S}\right]$, in which $C_{p}$ is the isobaric heat capacity (which in the data discussed below was measured with differential scanning calorimetry).

We focus the investigation on two well-studied van der Waals liquids, tetramethyl-tetraphenyl-trisiloxane (DC704) and 5-polyphenyl ether (5PPE), with values of $T_{g}$ at atmospheric pressure of 211 and $245 \mathrm{~K}$, respectively [33]. As an example, Fig. 1 collects the quantities needed to calculate $\gamma_{\tau}$ for DC704 from Eq. (7) where $K_{T}$ is computed from $K_{s}$ and $C_{p}$ as mentioned above. Figure 2(a) shows the dielectric relaxation times at atmospheric pressure for the silicone oil DC704, while Fig. 2(b) displays $\gamma_{\tau}$ at four temperatures along the $0.1 \mathrm{MPa}$ isobar. Figure 2(b) shows an increase of $\gamma_{\tau}$ as temperature increases or density decreases (inset) at ambient pressure. 

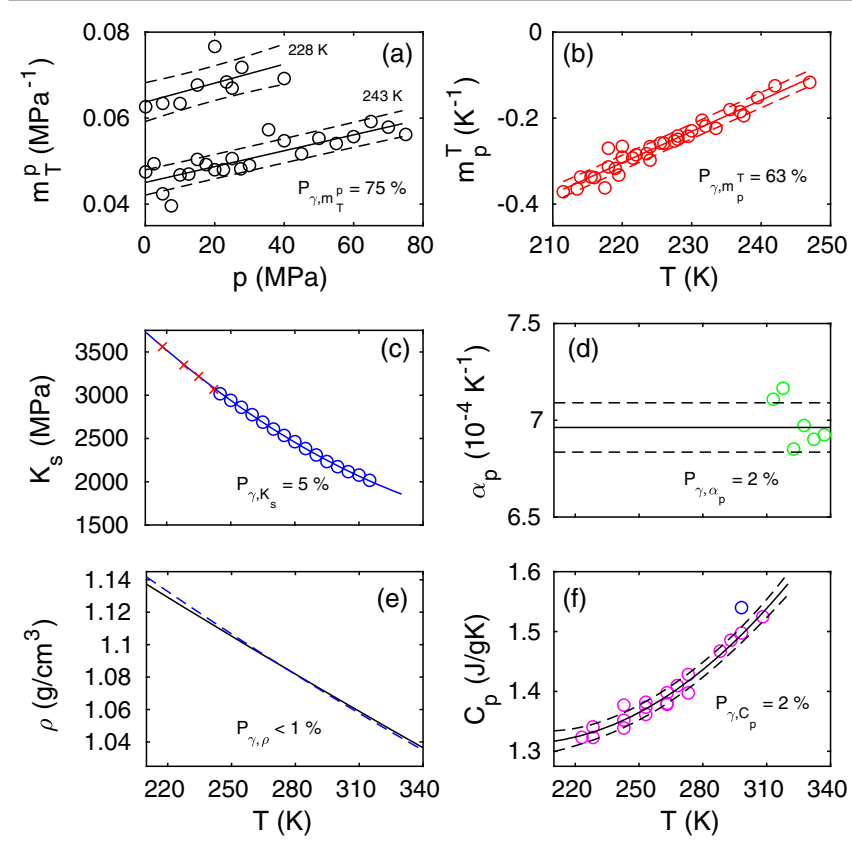

FIG. 1. Experimental dynamic and thermodynamic properties used for calculating the exponent $\gamma_{\tau}$ at two different state points, $(228 \mathrm{~K}, 0.1 \mathrm{MPa})$ and $(243 \mathrm{~K}, 0.1 \mathrm{MPa})$, for the silicone oil DC704. (a) The pressure fragility along two isotherms as a function of pressure (from dielectric relaxation measurements). The solid lines are estimated values and the dashed lines are the positive and negative uncertainties. (b) The temperature fragility at $0.1 \mathrm{MPa}$ as a function of temperature (from dielectric relaxation measurements). (c) Adiabatic bulk modulus from the standing waves in the piezoceramic bulk transducer [32]. Red crosses highlight the values of $K_{s}$ used for calculating $\gamma_{\tau}$ at four different temperatures. (d) The thermal expansion coefficient extracted from PVT measurements. (e) Temperature evolution of the density of liquid DC704 from the Tait equation (solid line) and from the extrapolation of the values at room conditions by using the expansion coefficient (dashed line). (f) Heat capacity as a function of temperature from differential scanning calorimetry measurements. The blue circle included in panel (f) corresponds to literature data [34]. The $P_{\gamma},{ }_{i}$ value in each panel indicates the contributions to the statistical error on the estimate of $\gamma_{\tau}$ at $T=228 \mathrm{~K}$ by using Eq. (6).

In order to evaluate to which extent the measured quantities contribute to the uncertainty of $\gamma_{\tau}$ and, in this way, to predict where one should give particular attention to reduce as much as possible the uncertainty, we use statistical tools. A large population of values for each variable $\left(N=10^{5}\right)$ are sampled by a Monte Carlo approach that assumes a Gaussian distribution centered about the mean with variance determined from the estimated error. The error is calculated by sampling a random collection of different scenarios for the variables in Eq. (7). The $P_{\gamma},{ }_{i}$ values in the panels of Fig. 1 represent the pairwise Pearson correlation coefficients between $\gamma_{\tau}$ and the variables involved in the computation of Eq. (7). If $P_{\gamma},{ }_{i}$ equals $100 \%$, there is a total positive correlation while zero
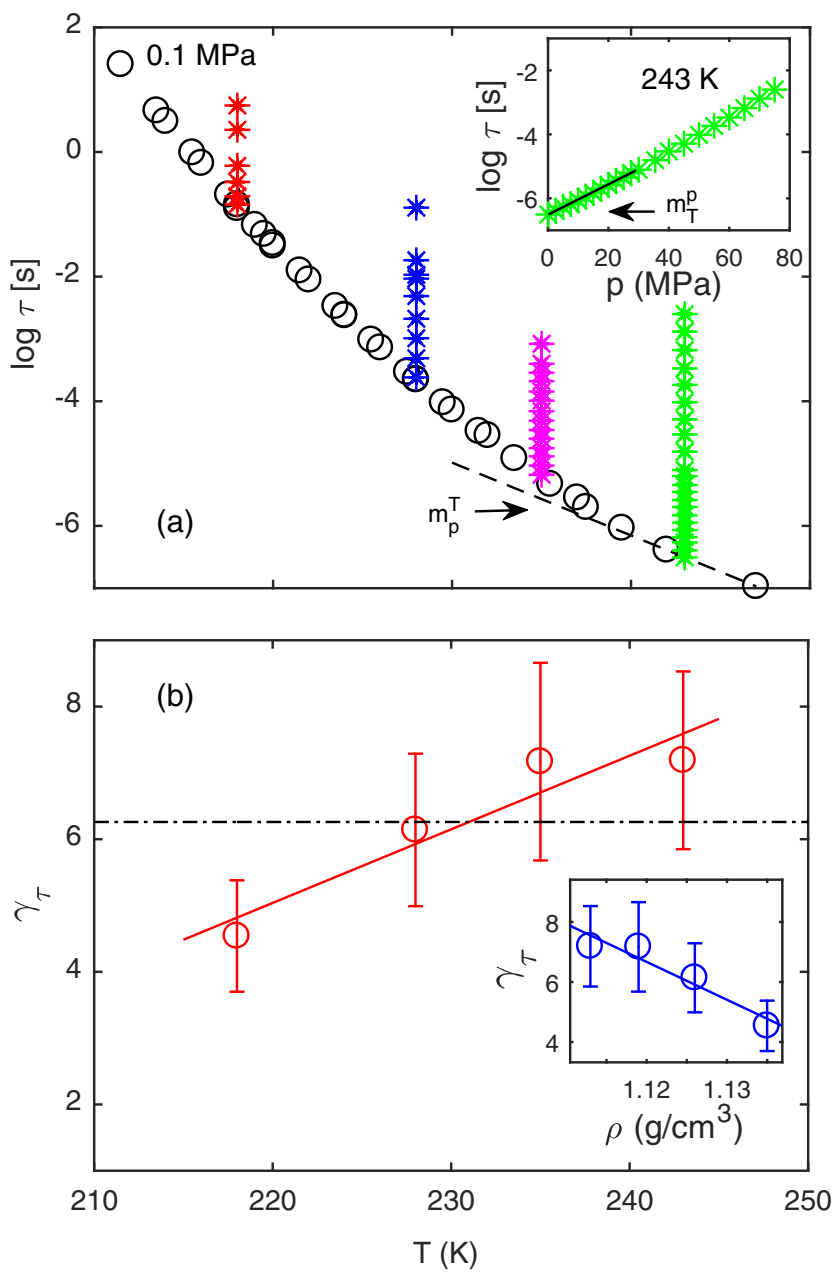

FIG. 2. (a) The structural relaxation time $\tau$ of DC704 determined from the dielectric loss-peak frequency. Black circles correspond to the temperature evolution at ambient pressure, while stars correspond to data at varying pressure; red 218, blue 228 , pink 235 , and green $242 \mathrm{~K}$. The inset shows the pressure dependence of the structural relaxation time for the isotherm at $243 \mathrm{~K}$, including the linear slope associated to the pressure fragility according to Eq. (3). These dielectric data have been acquired using a new home-built high-pressure setup and are in good agreement with previous data obtained by our group using different dielectric setups $[35,36]$. (b) Density-scaling exponent [Eq. (7)] as a function of temperature at atmospheric pressure (0.1 MPa). The black line indicates the average value of $\gamma_{\tau}$, the red line is a guide to the eye. The inset displays $\gamma_{\tau}$ as a function of density (the blue line is a guide to the eye).

indicates an absolute lack of correlation. We find that the properties with the strongest correlation with $\gamma_{\tau}$ are the pressure fragility $m_{T}^{p}$, followed by the temperature fragility $m_{p}^{T}$. It is therefore recommended to measure these two generalized fragilities with high accuracy in order to reduce the uncertainty in $\gamma_{\tau}$. On the other hand, slight variations in the density and in the thermal-expansion coefficient have only a minor effect on the resulting values of $\gamma_{\tau}$. The final error estimates on $\gamma_{\tau}$ are shown as error bars in Fig. 2(b). 
TABLE I. Density-scaling exponent of the structural relaxation time $\gamma_{\tau}$ at $p=0.1 \mathrm{MPa}$ for different substances obtained from Eq. (7).

\begin{tabular}{lcc}
\hline \hline Substance $\left(T_{g}\right)$ & $T$ & $\gamma_{\tau}$ \\
\hline DC704 $(211 \mathrm{~K})$ & $218 \mathrm{~K}$ & $4.5 \pm 0.8$ \\
& $228 \mathrm{~K}$ & $6.1 \pm 1.1$ \\
& $235 \mathrm{~K}$ & $7.1 \pm 1.5$ \\
5PPE $(245 \mathrm{~K})$ & $242 \mathrm{~K}$ & $7.2 \pm 1.3$ \\
Glycerol $(185 \mathrm{~K})$ & $268 \mathrm{~K}$ & $5.4 \pm 0.2$ \\
& $284 \mathrm{~K}$ & $7.8 \pm 0.5$ \\
DPG $(195 \mathrm{~K})$ & $230 \mathrm{~K}$ & $0.88 \pm 0.09$ \\
\hline \hline
\end{tabular}

Results are based on measurements presented in this study and data in Refs. [3,6,11,35,37-42].

Table I summarizes our findings for DC704 and 5PPE. The average $\gamma_{\tau}$ of DC704 is $6 \pm 1$ for the investigated state points. This is consistent with the density-scaling exponent 6.2 reported in Refs. [11,37] assuming a constant gamma (scenario A), suggesting that the exponent reported in Refs. [11,37] is the average of the actual, state-point dependent $\gamma_{\tau}$ 's. In Ref. [11] it was shown that $\gamma_{\tau}=\gamma_{S_{\mathrm{ex}}}$ for DC704. Thus, of the possible scenarios outlined above one concludes that DC704 belongs to either B or C. To demonstrate the general applicability of our approach, we include in Table I the values of $\gamma_{\tau}$ for the two hydrogenbonded liquids glycerol and dipropylene glycol (DPG) using own data, as well as data from the literature. Unlike the van der Waals bonded liquids DC704 and 5PPE, the hydrogen-bonded liquids have been shown to belong to scenario (D) with density-scaling exponents that depend on the observable [12]. The $\gamma_{\tau}$ values of the hydrogen-bonded liquids are much lower than those of the van der Waals bonded liquids. From the definition in Eq. (1) it follows that the dynamics of hydrogen-bonded liquids is less affected by a (relative) change of density than by a (relative) change of temperature.

Figure 3 shows that the density-scaling exponents are also state-point dependent for model liquids and metals. Figure 3(a) shows $\gamma_{S_{\mathrm{ex}}}$ computed from molecular dynamics simulations [43] of the Lennard-Jones (LJ) liquid [44] and of the Lennard-Jones trimer suggested by Lewis and Wahnström [45] as a coarse-grained model for orthoterphenyl (LW-oTP). In line with the experimental findings, the LW-oTP model shows an increase of the exponent with temperature, though less dramatic than in experiments (we note that this is an inaccurate model of real oTP, for instance, it predicts a wrong crystal structure [46]). In Fig. 3(b) we reanalyze experimental data for 20 metallic liquids [47], including metals where $a b$ initio density functional theory calculations demonstrate hidden-scale invariance [48]. For the monatomic metallic liquids, the density-scaling exponent of excess entropy is estimated using $\gamma_{S_{\mathrm{ex}}}=\left[\gamma_{G}-k_{B} / c_{v}\right] /\left[1-3 k_{B} / c_{v}\right]$, where $\gamma_{G}=\alpha_{p} K_{T} / \rho c_{v}$ is the thermodynamic Grüneisen parameter [49] (it is assumed that the material is above the Debye temperature $[13,48]$ ). The exponents have significant temperature dependencies with both positive and negative slopes along the $p=0.1 \mathrm{MPa}$ isobar. In Ref. [48] it was shown that the $a b$ initio energy surfaces of 57 elements have hidden-scale invariance. This suggests that metals belong to scenario $\mathrm{B}$ or $\mathrm{C}$. These results are in line with the state-point dependence of the densityscaling exponent found in simulations of the KobAndersen binary Lennard-Jones mixture [50], and they are also consistent with the generalized scaling equation of state reported in Ref. [51]. The latter study proposes a density dependence of the density-scaling exponent with two parameters that can be estimated from their generalized
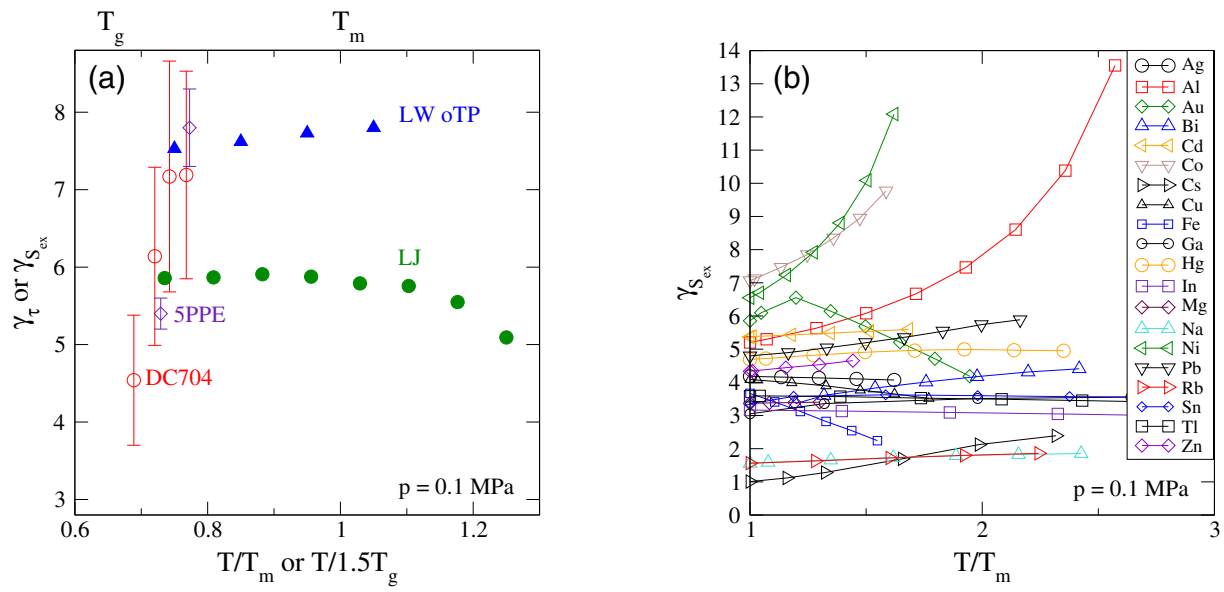

FIG. 3. (a) Comparison of experimentally determined density-scaling exponents (open symbols) with values of two model liquids (filled symbols). (b) The density-scaling exponent of the configurational adiabat $\gamma_{S_{\mathrm{ex}}}$ for liquid metals computed by reanalyzing experimental data compiled in Ref. [47]. 
density-scaling equation of state [51]. In this way, it is possible to determine the evolution of $\gamma$ with density from PVT measurements, as was recently reported for several organic liquids [52].

Finally, we note that Eq. (7) for $\gamma_{\tau}$ can be evaluated for any liquid regardless of its vitrification ability and that the formalism does not assume activated dynamics [21]. Thus, the suggested procedure can be used at any given point in the temperature-pressure plane. It has been shown that the isomorph theory allows one to make predictions for the variation of properties along the freezing line [53] and outof-equilibrium relaxation [54-56], assuming that a liquid belongs to scenarios $\mathrm{A}-\mathrm{C}$. For these applications it is pertinent to know $\gamma$ at the state points of interest.

In summary, we have shown that it is possible to determine the density-scaling exponent $\gamma_{\tau}$ from dynamic and thermodynamic properties of liquids. The analysis shows that $\gamma_{\tau}$ in general is state-point dependent. The expression presented in Eq. (7) connects $\gamma_{\tau}$ to measurable quantities that can be estimated over a wide range of thermodynamic conditions, from state points near the glass transition to well above the melting point (including elevated pressures). The new route is free of model assumptions.

This study was initiated after a series of discussions between Niels Boye Olsen and URP. This work is supported by the VILLUM Foundation's VKR-023455 and Matter (16515) Grants.

*asanz@ruc.dk

†urp@ruc.dk

[1] K. Binder and W. Kob, Glassy Materials and Disordered Solids: An Introduction to Their Statistical Mechanics (World Scientific, Singapore, 2005).

[2] C. Alba-Simionesco, A. Cailliaux, A. Alegria, and G. Tarjus, Scaling out the density dependence of the $\alpha$ relaxation in glass-forming polymers, Europhys. Lett. 68, 58 (2004).

[3] C. M. Roland, S. Hensel-Bielowka, M. Paluch, and R. Casalini, Supercooled dynamics of glass-forming liquids and polymers under hydrostatic pressure, Rep. Prog. Phys. 68, 1405 (2005).

[4] A. Tölle, H. Schober, J. Wuttke, O. G. Randl, and F. Fujara, Fast Relaxation in a Fragile Liquid under Pressure, Phys. Rev. Lett. 80, 2374 (1998).

[5] R. Casalini and C. M. Roland, Thermodynamical scaling of the glass transition dynamics, Phys. Rev. E 69, 062501 (2004).

[6] R. Casalini and C.M. Roland, Determination of the Thermodynamic Scaling Exponent for Relaxation in Liquids from Static Ambient-Pressure Quantities, Phys. Rev. Lett. 113, 085701 (2014).

[7] T. C. Ransom, M. Ahart, R. J. Hemley, and C. M. Roland, Vitrification and density scaling of polyurea at pressures up to $6 \mathrm{Gpa}$, Macromolecules 50, 8274 (2017).
[8] T. C. Ransom and W. F. Oliver, Glass Transition Temperature and Density Scaling in Cumene at Very High Pressure, Phys. Rev. Lett. 119, 025702 (2017).

[9] N. Gnan, T. B. Schrøder, U. R. Pedersen, N. P. Bailey, and J. C. Dyre, Pressure-energy correlations in liquids. IV. "Isomorphs" in liquid phase diagrams, J. Chem. Phys. 131, 234504 (2009).

[10] K. Niss, C. Dalle-Ferrier, G. Tarjus, and C. Alba-Simionesco, On the correlation between fragility and stretching in glass-forming liquids, J. Phys. Condens. Matter 19, 076102 (2007).

[11] D. Gundermann, U. R. Pedersen, T. Hecksher, N. P. Bailey, B. Jakobsen, T. Christensen, N. B. Olsen, T. B. Schroder, D. Fragiadakis, R. Casalini, C. M. Roland, J. C. Dyre, and K. Niss, Predicting the density-scaling exponent of a glassforming liquid from Prigogine-Defay ratio measurements, Nat. Phys. 7, 816 (2011).

[12] U. R. Pedersen, N. P. Bailey, T. B. Schrøder, and J. C. Dyre, Strong Pressure-Energy Correlations in van der Waals Liquids, Phys. Rev. Lett. 100, 015701 (2008).

[13] N. P. Bailey, U. R. Pedersen, N. Gnan, T. B. Schrøder, and J. C. Dyre, Pressure-energy correlations in liquids. II. Analysis and consequences, J. Chem. Phys. 129, 184508 (2008).

[14] J. C. Dyre, Hidden scale invariance in condensed matter, J. Phys. Chem. B 118, 10007 (2014).

[15] T. B. Schrøder and J. C. Dyre, Simplicity of condensed matter at its core: Generic definition of a Roskilde-simple system, J. Chem. Phys. 141, 204502 (2014).

[16] Y. Rosenfeld, Relation between the transport coefficients and the internal entropy of simple systems, Phys. Rev. A 15, 2545 (1977).

[17] J. Mittal, J. R. Errington, and T. M. Truskett, Relationship between thermodynamics and dynamics of supercooled liquids, J. Chem. Phys. 125, 076102 (2006).

[18] Y. Rosenfeld, A quasi-universal scaling law for atomic transport in simple fluids, J. Phys. Condens. Matter 11, 5415 (1999).

[19] N. Jakse and A. Pasturel, Excess entropy scaling law for diffusivity in liquid metals, Sci. Rep. 6, 20689 (2016).

[20] J. C. Dyre, Perspective: Excess-entropy scaling, J. Chem. Phys. 149, 210901 (2018).

[21] C. Alba-Simionesco, D. Kivelson, and G. Tarjus, Temperature, density, and pressure dependence of relaxation times in supercooled liquids, J. Chem. Phys. 116, 5033 (2002).

[22] C. Alba-Simionesco and G. Tarjus, Temperature versus density effects in glassforming liquids and polymers: A scaling hypothesis and its consequences, J. Non-Cryst. Solids 352, 4888 (2006).

[23] W. G. Hoover, S. G. Gray, and K. W. Johnson, Thermodynamic properties of the fluid and solid phases for inverse power potentials, J. Chem. Phys. 55, 1128 (1971).

[24] K. Koperwas, A. Grzybowski, K. Grzybowska, Z. Wojnarowska, J. Pionteck, A. P. Sokolov, and M. Paluch, Pressure coefficient of the glass transition temperature in the thermodynamic scaling regime, Phys. Rev. E 86, 041502 (2012).

[25] A. N. Papathanassiou, Density scaling of the diffusion coefficient at various pressures in viscous liquids, Phys. Rev. E 79, 032501 (2009). 
[26] M. Paluch, S. Haracz, A. Grzybowski, M. Mierzwa, J. Pionteck, A. Rivera-Calzada, and C. Leon, A Relationship between Intermolecular Potential, Thermodynamics, and Dynamic Scaling for a Supercooled Ionic Liquid, J. Phys. Chem. Lett. 1, 987 (2010).

[27] M. Romanini, M. Barrio, R. Macovez, M. D. Ruiz-Martin, S. Capaccioli, and J. L. Tamarit, Thermodynamic scaling of the dynamics of a strongly hydrogen-bonded glass-former, Sci. Rep. 7, 1346 (2017).

[28] C. A. Angell, Relaxations in Complex Systems (NRL, Washington, 1985).

[29] C. A. Angell, Formation of glasses from liquids and biopolymers, Science 267, 1924 (1995).

[30] G. Tarjus and C. Alba-Simionesco, An assessment of the concept of fragility, in Fragility of Glass-Forming Liquids (Hindustan Book Agency, India, 2014).

[31] C. A. Angell, Structural instability and relaxation in liquid and glassy phases near the fragile liquid limit, J. Non-Cryst. Solids 102, 205 (1988).

[32] T. Christensen and N.B. Olsen, Determination of the frequency-dependent bulk modulus of glycerol using a piezoelectric spherical shell, Phys. Rev. B 49, 15396 (1994).

[33] L. A. Roed, D. Gundermann, J. C. Dyre, and K. Niss, Communication: Two measures of isochronal superposition, J. Chem. Phys. 139, 101101 (2013).

[34] I. S. Klein and C. A. Angell, Excess thermodynamic properties of glassforming liquids: The rational scaling of heat capacities, and the thermodynamic fragility dilemma resolved, J. Non-Cryst. Solids 451, 116 (2016).

[35] D. Gundermann, Testing predictions of the isomorph theory by experiment, $\mathrm{Ph}$. D. thesis, IMFUFA, Roskilde University, 2013.

[36] B. Jakobsen, K. Niss, and N. B. Olsen, Dielectric and shear mechanical alpha and beta relaxations in seven glassforming liquids, J. Chem. Phys. 123, 234511 (2005).

[37] R. Casalini, R. F. Gamache, and C. M. Roland, Densityscaling and the Prigogine-Defay ratio in liquids, J. Chem. Phys. 135, 224501 (2011).

[38] K. Adrjanowicz, K. Kaminski, M. Tarnacka, G. Szklarz, and M. Paluch, Predicting nanoscale dynamics of a glassforming liquid from its macroscopic bulk behavior and vice versa, J. Phys. Chem. Lett. 8, 696 (2017).

[39] C. Dreyfus, A. Le Grand, J. Gapinski, W. Steffen, and A. Patkowski, Scaling the $\alpha$-relaxation time of supercooled fragile organic liquids, Eur. Phys. J. B 42, 309 (2004).

[40] C. Klieber, T. Hecksher, T. Pezeril, D. H. Torchinsky, J. C. Dyre, and K. A. Nelson, Mechanical spectra of glass-forming liquids. II. Gigahertz-frequency longitudinal and shear acoustic dynamics in glycerol and DC704 studied by time-domain Brillouin scattering, J. Chem. Phys. 138, 12A544 (2013).

[41] A. Grzybowski, K. Grzybowska, J. Zioło, and M. Paluch, Correlations between isobaric and isochoric fragilities and thermodynamical scaling exponent for glass-forming liquids, Phys. Rev. E 74, 041503 (2006).

[42] A. Sanz, Density scaling in dipropylene glycol by dielectric spectroscopy measurements in pressure-temperature plane (unpublished).

[43] N. P. Bailey, T. S. Ingebrigtsen, J. S. Hansen, A. A. Veldhorst, L. Bøhling, C. A. Lemarchand, A. E. Olsen, A. K. Bacher, L. Costigliola, U. R. Pedersen, H. Larsen, J. C. Dyre, and T. B. Schrøder, RUMD: A general purpose molecular dynamics package optimized to utilize GPU hardware down to a few thousand particles, SciPost Phys. 3, 038 (2017).

[44] J. E. Lennard-Jones, On the determination of molecular fields.-I. From the variation of the viscosity of a gas with temperature, Proc. R. Soc. A 106, 441 (1924).

[45] L. J. Lewis and G. Wahnström, Relaxation of a molecular glass at intermediate times, Solid State Commun. 86, 295 (1993).

[46] U. R. Pedersen, T. S. Hudson, and P. Harrowell, Crystallization of the Lewis-Wahnström ortho-terphenyl model, J. Chem. Phys. 134, 114501 (2011).

[47] R. N. Singh, S. Arafin, and A. K. George, Temperaturedependent thermo-elastic properties of s-, p- and d-block liquid metals, Physica (Amsterdam) 387B, 344 (2007).

[48] F. Hummel, G. Kresse, J. C. Dyre, and U. R. Pedersen, Hidden scale invariance of metals, Phys. Rev. B 92, 174116 (2015).

[49] E. Grüneisen, Theorie des festen Zustandes einatomiger Elemente, Ann. Phys. (Berlin) 344, 257 (1912).

[50] L. Bøhling, T. S. Ingebrigtsen, A. Grzybowski, M. Paluch, J. C. Dyre, and T. B. Schrøder, Scaling of viscous dynamics in simple liquids: theory, simulation and experiment, New J. Phys. 14, 113035 (2012).

[51] A. Grzybowski, K. Koperwas, and M. Paluch, Equation of state in the generalized density scaling regime studied from ambient to ultra-high pressure conditions, J. Chem. Phys. 140, 044502 (2014).

[52] E. R. López, O. Fandino, D. Cabaleiro, L. Lugo, and J. Fernández, Determination of derived volumetric properties and heat capacities at high pressures using two density scaling based equations of state. Application to dipentaerythritol hexa(3,5,5- trimethylhexanoate), Phys. Chem. Chem. Phys. 20, 3531 (2018).

[53] U. R. Pedersen, L. Costigliola, N. P. Bailey, T. B. Schrøder, and J.C. Dyre, Thermodynamics of freezing and melting, Nat. Commun. 7, 12386 (2016).

[54] N. Gnan, C. Maggi, T. B. Schrøder, and J. C. Dyre, Predicting the Effective Temperature of a Glass, Phys. Rev. Lett. 104, 125902 (2010).

[55] K. Niss, Mapping Isobaric Aging onto the Equilibrium Phase Diagram, Phys. Rev. Lett. 119, 115703 (2017).

[56] J. C. Dyre, Isomorph theory of physical aging, J. Chem. Phys. 148, 154502 (2018) 